\title{
MANEJO DE LA PREECLAMPSIA / ECLAMPSIA EN EL INSTITUTO NACIONAL MATERNO PERINATAL
}

\author{
Enrique Guevara Ríos ${ }^{1}$ \& Luis Meza Santibañez ${ }^{2}$.
}

\begin{abstract}
RESUMEN
La preeclampsia es una enfermedad de carácter progresiva e irreversible que afecta múltiples órganos y debido a que lesiona a la madre y al feto, es responsable de una proporción considerable de muertes maternas y perinatales. Complica el 3 al $22 \%$ de los embarazos y es la segunda causa de muerte materna en el Perú en el 2014 con el 38\%, y la primera causa de muerte materna en el Instituto Nacional Materno Perinatal con el $43 \%$. El espectro clínico de la preeclampsia varía desde formas leves a severas. Los criterios de diagnóstico de preeclampsia son hipertensión y proteinuria, sin embargo la proteínuria mayor de 5 grs ya no se considera como un criterio de preeclampsia severa. Se hace una revisión de los nuevos aportes en el manejo de la preeclampsia severa y eclampsia y en el manejo de una de sus complicaciones más graves como es la rotura hepática.
\end{abstract}

Palabras clave: Preeclampsia; Eclampsia; Sindrome HELLP (Fuente: DeCS BIREME).

\section{MANAGEMENT OF PREECLAMPSIA / ECLAMPSIA AT THE NATIONAL MATERNAL PERINATAL INSTITUTE}

\begin{abstract}
Preeclampsia is a progressive and irreversible disease that affects multiple organs and because it will harm the mother and fetus, is responsible for a considerable proportion of maternal and perinatal deaths. Complicates 3 to $22 \%$ of pregnancies and is the second leading cause of maternal death in Peru 2013 with $38 \%$, and the leading cause of maternal death in the National Maternal Perinatal Institute with $43 \%$. The clinical spectrum of preeclampsia ranges from mild to severe forms. The diagnostic criteria of preeclampsia are hypertension and proteinuria, however most Proteinuria $5 \mathrm{~g}$ no longer considered as a criterion of severe preeclampsia. Carried out a review of the new contributions in the management of severe preeclampsia and eclampsia and the management of one of its most serious complications sucha as the liver rupture.
\end{abstract}

Key words: Preeclampsia; eclampsia; HELLP syndrome (Source: MeSH NLM).

\section{INTRODUCCIÓN}

La preclampsia es una enfermedad de carácter progresiva e irreversible que afecta múltiples órganos ${ }^{1} y$, debido a que lesiona a la madre y al feto, es responsable de una proporción considerable de muertes maternas y perinatales. Complica del 3 al $22 \%$ de los embarazos ${ }^{2}$. En el Perú su incidencia fluctúa entre el 10 al $15 \%$ en la población hospitalaria ${ }^{3}$. La incidencia de la enfermedad hipertensiva del embarazo es mayor en la costa que en la sierra, pero la mortalidad materna por esta causa es mayor en la sierra; no parece existir diferencias mayores entre las características de esta enfermedad en ambas regiones ${ }^{4}$. Es la segunda causa de muerte materna en el Perú en el 2013 con un $38 \%{ }^{5}$. A nivel de la Dirección de Salud de Lima Ciudad es la primera causa de muerte entre los años 2000 al 2009 con un 33\%.

En el Instituto Nacional Materno Perinatal es la primera causa de muerte materna entre los años 2003 y 2013 con un $43 \% 5$. Durante el 2013 se atendieron un total de 19,950 egreso obstétricos, de los cuales estuvieron complicados con problemas de hipertensión arterial, 1,611 casos $(8,1 \%)$ de estos casos 15,522 fueron preeclampsia (94,5\%), 24 casos de eclampsia $(1,5 \%)^{6}$. Durante ese mismo período se presentaron 65 (4\%) casos de Síndrome de HELLP.

En el 2014 se atendieron un total de 21,079 egreso obstétricos, de los cuales estuvieron complicados con problemas de hipertensión arterial, 1,197 casos $(5.6 \%)$ de estos casos 1,136 fueron preeclampsia (94.9\%), 23 casos de eclampsia $(1.9 \%)^{6}$. Durante ese mismo período se presentaron 38 (3.2\%) casos de Síndrome de HELLP. En el 2013 en 9 gestantes (1\%) complicadas con preeclampsia/eclampsia, el parto se produjo antes de las 28 semanas de gestación; en 165 gestantes (17\%), el parto se produjo entre las 28 y 34 semanas; y en 801 gestantes $(82 \%)$ el parto se produjo después de las 34 semanas.

Médico Gineco-Obstetra, Director del Instituto Nacional Materno Perinatal. Docente de la Facultad de Medicina de la Universidad Nacional Mayor de San Marcos. Miembro Asociado de la Sociedad Peruana de Obstetricia y Ginecología. Lima-Perú.

2 Médico Gineco-Obstetra, Jefe de la Unidad de Docencia de la Oficina Ejecutiva de Docencia e Investigación del Instituto Nacional Materno Perinatal. Docente de la Facultad de Medicina de la Universidad Nacional Mayor de San Marcos. Miembro Titular de la Sociedad Peruana de Obstetricia y Ginecología. 


\begin{tabular}{|c|c|c|c|c|}
\hline \multirow{2}{*}{ DIAGNOSTICO PRINCIPAL DE EGRESO OBSTETRICO } & \multicolumn{2}{|c|}{2,013} & \multicolumn{2}{|c|}{2,014} \\
\hline & $\mathrm{N}^{*}$ & $\%$ & $\mathrm{~N}^{*}$ & $\%$ \\
\hline TRANSTORNOS HIPERTENSIVO DEL EMBARAZO & 1,611 & 8.1 & 1,197 & 5.6 \\
\hline PRE ECLAMPSIA & 1,522 & 94.5 & 1,136 & 94.9 \\
\hline ECLAMPSIA & 24 & 1.5 & 23 & 1.9 \\
\hline SINDROME DE HELLP & 65 & 4.0 & 38 & 3.2 \\
\hline HEMORRAGIA OBSTETRICA & 589 & 3.0 & 875 & 4.1 \\
\hline DESPREND, PREMATURO DE PLACENTA & 90 & 15.3 & 144 & 16.5 \\
\hline PLACENTA PREVIA & 143 & 24.3 & 204 & 23.3 \\
\hline RUPTURA UTERINA & 26 & 4.4 & 19 & 2.2 \\
\hline HEMORRAGIA POST PARTO & 319 & 54.2 & 491 & 56.1 \\
\hline SHOCK HIPOVULEMICO & 11 & 1.9 & 17 & 1.9 \\
\hline
\end{tabular}

Fuente: Oficina de Estadística e Informática INMP- 2015

De las pacientes con preeclampsia/eclampsia, el parto vaginal se produjo en 186 casos (19\%) y el parto fue por cesárea en 789 casos (81\%).

Se hospitalizaron 449 recién nacidos (46\%) del total de gestantes con preeclampsia/eclampsia, lo cual evidencia el riesgo alto de morbilidad neonatal por razones de esta patología. 75 recién nacidos $(17 \%)$ fueron pequeños para edad gestacional y $330(73 \%)$ fueron adecuados para la edad gestacional.

\section{DIAGNÓSTICO DE PREECLAMPSIA}

El espectro clínico de la preeclampsia varía desde formas leves a severas. En la mayoría de las mujeres, la progresión a través de este espectro es lento, y el diagnóstico de preeclampsia leve debe ser interpretado como una fase de la enfermedad ${ }^{1}$. En otros, la enfermedad progresa más rápidamente, cambiando de leves a graves en días o semanas. En los casos más graves, la progresión puede ser fulminante, con evolución de preeclampsia leve a preeclampsia severa o eclampsia en cuestión de días o incluso horas. Por lo tanto, para el manejo clínico, la preeclampsia se debe sobrediagnosticar, debido a que el objetivo más importante en el manejo de la preeclampsia es la prevención de la morbilidad y mortalidad materna y perinatal.

Los criterios de diagnóstico de preeclampsia son hipertensión y proteinuria ${ }^{1,7,8,9,10}$.

La hipertensión es definida como la presión arterial sistólica igual o mayor de $140 \mathrm{~mm} \mathrm{Hg}$ o diastólica igual o mayor de $90 \mathrm{~mm} \mathrm{Hg}$ tomadas en dos ocasiones y con un intervalo de 4 horas, y que ocurre después de las 20 semanas de embarazo en mujeres con presión arterial previa normal; o presión arterial sistólica igual o mayor de $160 \mathrm{~mm} \mathrm{Hg}$ o diastólica igual o mayor de $110 \mathrm{~mm}$ de $\mathrm{Hg}$ 1,7,8,9,10. La elevación de $30 \mathrm{~mm} \mathrm{Hg}$ en la presión sistólica o de $15 \mathrm{~mm} \mathrm{Hg}$ en la diastólica respecto a la presión basal es un signo de alerta y requiere una vigilancia muy estrecha, especialmente si está presente la proteinuria.
La medición de la presión arterial debe hacerse con la gestante sentada por lo menos tres minutos antes de su realización y debe usarse un brazalete adecuado para el diámetro del brazo teniendo ${ }^{11}$ : Se debe palpar la arteria braquial e insuflar el balón $20 \mathrm{~mm} \mathrm{Hg}$ por encima del nivel en que se deja de palpar el pulso. El brazalete debe desinflarse lentamente (2 $\mathrm{mm} \mathrm{Hg}$ por segundo). El valor sistólico debe observarse directamente en el esfigmomanómetro y el diastólico por auscultación del $5^{\circ}$ ruido de Korotkoff. Solo se utilizará el $4^{\circ}$ ruido de Korotkoff cuando el $5^{\circ}$ se encuentre muy próximo al cero.

En la primera consulta debe hacerse la medición en ambos brazos y en posteriores controles en el brazo derecho. Si hay diferencia significativa en ambos brazos debe enviarse a valoración especializada.

Se debe insistir en que las pacientes menores de 18 años fisiológicamente presentan cifras tensionales menores que las mujeres adultas, por lo tanto el límite patológico del 140/90 mm Hg no es extrapolable a esta población.

No es indispensable el uso de equipos electrónicos de manera rutinaria para medir la presión arterial. Los tensiómetros de columna de mercurio siguen siendo la mejor opción como instrumento para medir la tensión arterial, por lo tanto, se recomienda como elemento importante en los consultorios de maternidad y en las salas de admisiones para trabajo de parto.

La proteinuria definida como presencia de proteínas en orina de 24 horas $\geq 300 \mathrm{mg}^{1,2,5,7-10}$ presencia de proteínas de $2+$ al usar tira reactiva o de $1+$ con ácido sulfosalicílico (ASS) en 2 muestras de orina tomada al azar con por lo menos 4 horas de diferencia 1,15,38; o un cociente de proteína urinaria / creatinina urinaria $\geq 0,3$ 22,29. Sospecha de proteinuria: Presencia de proteínas de $1+$ al usar tira reactiva o trazas con el ácido sulfosalicílico (ASS) en orina tomada al azar. En caso de sospecha repetir a las 4 horas y realizar proteinuria de 24 horas.

En vista de que recientes investigaciones no han encontrado relación entre la cantidad de proteínas en 
orina y el pronóstico de la preeclampsia, la proteínuria mayor de 5 grs ya no se considera como un criterio de preeclampsia severa ${ }^{1,8}$.

En el Instituto Nacional Materno Perinatal se realiza la prueba con ácido sulfosalicílico (ASS) al 3\%, que se realiza mezclando $1 \mathrm{cc}$ de ASS, conservado en frasco oscuro con $1 \mathrm{cc}$ de orina centrifugada, o decantada 1 hora, tomar muestra del sobrenadante; se mezcla por inversión y dejar reposar por 5 minutos y luego se realizar lectura ${ }^{12}$

\begin{tabular}{|c|c|c|}
\hline & Proteinuria & Característica \\
\hline NEGATIVO & $0 \mathrm{mg}$ por $100 \mathrm{cc}$ & Transparente \\
\hline TRAZAS & $<50 \mathrm{mg}$ por $100 \mathrm{cc}$ & Turbidez perceptible \\
\hline $1+$ & 50 mg. por $100 \mathrm{cc}$ & $\begin{array}{l}\text { Turbidez sin } \\
\text { granulación }\end{array}$ \\
\hline $2+$ & $200 \mathrm{mg}$. por $100 \mathrm{cc}$ & $\begin{array}{l}\text { Turbidez con } \\
\text { granulación }\end{array}$ \\
\hline $3+$ & $500 \mathrm{mg}$. por $100 \mathrm{cc}$ & $\begin{array}{l}\text { Turbidez con } \\
\text { granulación y } \\
\text { aglutinación }\end{array}$ \\
\hline $4+$ & 1000 mg. Por $100 \mathrm{cc}$ & Precipitado solido \\
\hline
\end{tabular}

Existe gran discrepancia para la cuantificación de la proteinuria al utilizar muestra aislada de orina, dependiendo del fabricante y de la sensibilidad química del reactivo utilizado en las tiras. La muestra aislada presenta una tasa alta de falsos negativos, en razón que la excreción de proteinas no es en forma constante con cada micción, es por pulsos, por lo cual se ha tratado de recomendar por su versatilidad la relación proteinuria/creatinuria, para evitar la necesidad de realizar recolecciones de orina de 24 horas $^{13}$

El valor clásico de $300 \mathrm{mg}$ de proteína en orina de 24 horas es obtenido de estudios de mala calidad. El único estudio realizado para obtener un punto de corte preciso que discrimine la anormalidad, encontró ésta en $200 \mathrm{mg}$ en la orina de 24 horas. Sin embargo, se seguirá utilizando el valor más tradicional y universal de $300 \mathrm{mg}$ en orina de 24 horas $^{14}$.

El edema no se incluye en el diagnóstico de preeclampsia por su frecuente aparición clínica, hasta en $80 \%$ de las gestantes normales, razón por la cual este signo ha sido abolido en los consensos internacionales para el diagnóstico de preeclampsia ${ }^{15}$. Sin embargo, en nuestro medio es conveniente considerar los edemas patológicos de rápida instauración confirmados por ganancias ponderales anormales (ganancia de peso materno mayor a $800 \mathrm{~g} /$ semana) como un signo útil para el diagnóstico temprano de la entidad ${ }^{15}$. En la calificación del edema se tiene un edema fisiológico de la gestante que se encuentra a nivel del tercio inferior de miembros inferiores, este edema cede con el reposo y elevación de miembros inferiores, todo edema por encima se considera patológico. Consideramos $(+)$ cuando compromete miembros inferiores, $(++)$ cuando el edema llega a abdomen y manos, $(+++)$ el edema llega hasta la cara, $(++++)$ presenta anasarca, ascitis, derrame pericárdico, derrame pleural, este edema podría estar acompañando a la pre eclampsia severa.

\section{CLASIFICACIÓN DE LA PREECLAMPSIA}

La preeclampsia se clasifica en preeclampsia leve, preeclampsia severa y eclampsia. La preeclampsia leve presenta una presión arterial mayor de 140/90 mm $\mathrm{Hg}$ pero menor de $160 / 110 \mathrm{~mm} \mathrm{Hg}$, con presencia de proteinuria; y la preeclampsia severa presenta una presión arterial igual o mayor de 160/110 mm Hg. La eclampsia es definida como la presencia de convulsiones que no pueden ser atribuídas a otras causas en una gestante con preeclampsia $1,9,10$

En muchos casos se presentan gestantes con hipertensión sin proteinuria que pueden ser diagnosticadas de preeclampsia severa si presentan alguno de los siguientes criterios $^{1}$ : Presión arterial sistólica $\geq 160$ y diastólica $\geq$ $110 \mathrm{mmHg}$ tomadas en por lo menos dos ocasiones y con un intervalo de 4 horas; trombocitopenia con un recuento de plaquetas menor de 100,000/ microlitro; aumento de enzimas transaminasas hepáticas al doble de los valores normales; insuficienia renal progresiva con una concentración de creatinina mayor de $1.1 \mathrm{mg} / \mathrm{dl}$ o un aumento del doble en ausencia de una enfermedad renal; edema pulmonar; síntomas cerebrales o visuales; o dolor severo o persistente en el hipocondrio derecho o en epigastrio. En el último reporte del Colegio Americano de Obstetras y Ginecólogos de noviembre del 2013, ya no consideran como criterio de preeclampsia severa la restricción del crecimiento intrauterino y el oligoamnios ${ }^{1}$.

\section{MANEJO DE LA ENFERMEDAD HIPERTENSIVA DEL EMBARAZO:}

\section{A.- MANEJO EN CONSULTORIO EXTERNO:}

\section{Gestante de riesgo para desarrollar trastorno hipertensivo debe ser derivado a consultorio de Obstetricia "C":}

a) IP de las arterias uterinas > p95 en las ecografías doppler de las 11 a 13 semanas o de las 20 a 24 semanas.

b) Elevación de la presión arterial: aumento de la sistólica en $30 \mathrm{mmHg}$ o de la diastólica en $15 \mathrm{mmHg}$ respecto a la presión basal. Si PA es $\geq 140 / 90 \mathrm{mmHg}$ debe pasar al servicio de Emergencia para realizar estudios.

c) Ganancia de peso $>500 \mathrm{gr}$ por semana en el tercer trimestre.

d) Acido Sulfo Salicílico positivo.

\section{En cada atención prenatal de una paciente de alto} riesgo se debe realizar:

a) Llenado de las gráficas de ganancia de peso y altura uterina.

b) Prueba de ASS en cada consulta a partir de las 20 semanas de gestación.

c) Ecografías doppler de las arterias uterinas entre 
las 11 a 13 semanas y entre las 20 a 24 semanas.

d) Entre las 28 y 32 semanas solicitar los exámenes de laboratorio antes descrito.

3. Medidas preventivas.- Las cuales se debe realizar en pacientes de alto riesgo:

a) Ácido acetil salicílico: Se recomienda la administración oral de 75 a $150 \mathrm{mg}$ de aspirina todos los días a partir de las 12 semanas ( $y$ antes de las 16 semanas) ${ }^{15,24-28,37}$ hasta las 34 semanas de gestación. Si la paciente llega a hacer preeclampsia severa antes de las 34 semanas: suspender la aspirina (idealmente 5 a 10 días antes de la culminación del embarazo).

b) Administración de calcio: Se recomienda la ingesta de 1,5 a $2 \mathrm{gr}$ de calcio por día a partir de las 14 semanas de gestación ${ }^{15}$.

\section{Criterios para el manejo ambulatorio de trastornos hipertensivos:}

a) Presión arterial sistólica no mayor de $140 \mathrm{mmHg}$ y/o diastólica no mayor de $90 \mathrm{mmHg}$.

b) Proteinuria $<300 \mathrm{mg}$ en orina de 24 horas.

c) Ausencia de síntomas de preeclampsia.

d) Recuento plaquetario $>150,000 / \mathrm{mm} 3$.

e) Ausencia de daño de órgano blanco.

f) Bienestar fetal conservado.

En todas las pacientes con manejo ambulatorio debe comprobarse el fácil acceso al sistema de salud y la comprensión de los signos de alarma.

El control prenatal se realizará a los 7 días, con medición diaria de PA, detectando signos o síntomas de severidad en forma temprana; y con evaluaciones de medicina fetal, cardiología, oftalmología, endocrinología y nutrición según sea el caso.

En gestantes con hipertensión arterial crónica dar alfa metildopa a dosis terapéuticas para la hipertensión arterial sin bajar la presión diastólica menos de $90 \mathrm{mmHg}$, y se debe realizar controles cada 15 días hasta las 32 semanas y luego en forma semanal.

\section{MANEJO DE LA PREECLAMPSIA}

Establecido el diagnóstico de preeclampsia, se requiere inmediatamente evaluar el estado de salud de la gestante y el feto. Se debe realizar una evaluación clínica de las funciones neurológica, respiratoria y cardiovascular; al mismo tiempo se debe realizar una evaluación hematológica y bioquímica del número de plaquetas, pruebas de función renal (urea, creatinina, y ácido úrico), y pruebas de función hepática (transaminasas y lactato deshidrogenasa) 1,9,10,11. Se debe evaluar el bienestar fetal con el Monitoreo Fetal Electrónico: test no estresante o estresante, con el Perfil biofísico, y con el estudio Doppler de las arterias umbilical y uterina. Si se considere necesaria la valoración del grado de madurez pulmonar fetal ${ }^{20}$ se realizará la amniocentesis.

La gestante con preeclampsia leve, severa o eclampsia requiere hospitalización inmediata para su manejo respectivo ${ }^{16}$, con el objetivo terapéutico ${ }^{10,16}$ de controlar la hipertensión arterial, prevenir la ocurrencia de convulsiones o su repetición, optimizar el volumen intravascular, mantener una oxigenación arterial adecuada y diagnosticar y tratar precozmente las complicaciones.

En la preeclampsia el tratamiento final es la interrupción del embarazo y generalmente se toma en cuenta las condiciones maternas para dicha interrupción, antes que la edad gestacional y la salud fetal.

\section{MANEJO GENERAL DE LA PREECLAMPSIA LEVE, SEVERA O ECLAMPSIA:}

Toda paciente con diagnóstico de preeclampsia leve, severa o con eclampsia debe ser hospitalizada en un establecimiento de II-2 o III nivel de atención, de preferencia en un establecimiento que disponga de una Unidad de Cuidados Intensivos Materna, Unidad de Cuidados Intensivos Neonatal y Banco de Sangre. Durante la hospitalización se debe monitorizar la presión arterial cada 4 horas, así como las funciones vitales, latidos cardiacos fetales y contracciones uterinas; del mismo modo se debe controlar la diuresis y realizar el control bioquímico y hematológico: perfil de coagulación, perfil renal: creatinina, úrea y ácido úrico, depuración de creatinina; proteinuria cualitativa diaria, proteinuria cuantitativa semanal, control diario de peso, balance de líquidos administrados y eliminados, perfil hepático: enzimas hepáticas; diario o más seguido de ser necesario. Se debe evaluar el bienestar fetal por lo menos cada 72 horas

\section{1.- Medidas generales:}

- Dieta normo proteica y normo sódica.

- Monitoreo de la presión arterial cada 4 horas en hoja aparte, control de peso y diuresis diaria; y detección precoz de signos y síntomas de alarma.

- Solicitar exámenes de laboratorio para descartar compromiso de órgano blanco, los cuales deben ser repetidos según la evolución de la paciente.

2.- Medidas específicas de la Hipertensión Gestacional:

- Solicitar pruebas de bienestar fetal cada semana:

- Ecografía Doppler y perfil biofísico.

- Monitoreo Electrónico Fetal: NST y/o TS, según el caso.

- No usar antihipertensivos en forma horaria.

- Culminar el embarazo en gestaciones $\geq 37$ semanas. 


\section{MANEJO DE LA PREECLAMPSIA LEVE}

Se requiere de un reposo relativo ${ }^{1,17,18,19}$ y de un dieta normo calórica, normo proteica y normosódica ${ }^{17}$. No está indicado el uso de antihipertensivos ${ }^{1,17,18,19}$. El tratamiento definitivo de la preeclampsia es culminar la gestación. Si la gestante tiene 37 semanas o más, se debe culminar la gestación por vía vaginal o por cesárea 1,17,18,19,20,21. Si la gestante tiene menos de 37 semanas se puede tener una conducta expectante si no hay afectación materna o fetal. Durante el manejo expectante se debe continuar con las medidas general y una estricta vigilancia materna y fetal ${ }^{18,19,20,21}$. Si a pesar del tratamiento no se controla la hipertensión arterial, o la enfermedad progresa a preeclampsia severa, o existen pródromos de eclampsia, o existen signos de sufrimiento fetal, o existen signos de disfunción hematológica, renal, hepática, pulmonar o neurológia, se debe finalizarse la gestación inmediatamente independiente de la edad gestacional; ya sea por vía vaginal o por cesárea1, 10,18,19,20,21

\section{MANEJO DE LA PREECLAMPSIA SEVERA}

La preeclampsia severa evoluciona hacia complicaciones graves como edema agudo de pulmón, falla renal, encefalopatía hipertensiva con hemorragia cerebral, desprendimiento de retina, desprendimiento prematuro de placenta, hematoma subcapsular hepático o rotura hepática, sindrome HELLP, que pueden llevar a la muerte de la gestante y del feto o recién nacido ${ }^{1,17,18,19,20,21}$. Por lo tanto el manejo debe ser oportuno, multidisciplinario y efectivo ${ }^{8,15,21,23}$ : Se debe sospechar de preeclampsia en una gestante que presenta sangrado vaginal y signos de desprendimiento prematuro de placenta.

En la preeclampsia hay una contracción del intravascular, a diferencia de la hipervolemia usual de la gestante; por lo tanto hay tendencia a oliguria por falta de fluidos, a pesar del edema. Deberá asegurarse una expansión adecuada del intravascular con solución salina al 9 o/oo .

- Hidratación: abrir una vía venosa periférica con un catéter $\mathrm{N}^{\circ} 18$ con $\mathrm{NaCl} 90 / 00$, y pasar 1000 cc a chorro en 20 minutos, luego de lo cual se evaluará el estado de hidratación de la paciente; si se evidencia signos de deshidratación (FC >90 x', diuresis $<30 \mathrm{cc} /$ hora, mucosa oral seca) se continuará la hidratación con reto de fluidos.

- Uso de anticonvulsivante: Iniciar por otra vía venosa una infusión de Sulfato de Magnesio: diluir 5 ampollas de $504 \mathrm{Mg}$ al $20 \%$ en 50 cc de $\mathrm{NaCl} 90 / 00$ y pasar por volutrol, con bomba de infusión, $40 \mathrm{cc}(4 \mathrm{gr})$ en 20 minutos y continuar con $10 \mathrm{cc}(1 \mathrm{gr})$ por hora hasta 24 horas post-parto ${ }^{15}$. En caso de eclampsia se administrará $40 \mathrm{cc}$ (4gr) en 20 minutos y se continuará con $20 \mathrm{cc}$ (2 gr) por hora.
Durante la administración de SO4Mg se deberán realizar los siguientes controles:

- Reflejo rotuliano: Debe estar presente.

- Control de diuresis horaria: debe ser $>30 \mathrm{cc} / \mathrm{hora}$, caso contrario se suspende el SO4Mg.

- Frecuencia respiratoria: debe ser $>14$ respiraciones / minuto.

- Frecuencia cardiaca: debe ser $>60 \mathrm{x}$ '.

- Control de la saturación de O2 mediante pulsioximetría.

o Control estricto de funciones vitales cada hora en hoja aparte.

- Si se detecta arreflexia tendinosa suspender la administración de Sulfato de Magnesio.

- Si hay depresión del estado de conciencia, tendencia a la ventilación superficial o lenta (FR $<14$ por minuto) debe administrarse Gluconato de Calcio al $10 \%$, una ampolla de $10 \mathrm{ml}$ endovenoso en un periodo de 10 minutos $^{37}$.

- Administrar en forma prudente el SO4Mg en casos de insuficiencia renal aguda.

- Uso de antihipertensivos:

- Metildopa: administrar $1 \mathrm{gr}$ VO cada 12 horas apenas se realice el diagnóstico de preeclampsia severa.

- Nifedipino: Si luego de 20 minutos de iniciado el manejo continua la paciente con hipertensión severa, presión arterial mayor de 160/110, administrar nifedipino $10 \mathrm{mg}$ VO y, si es necesario, repetir cada 20 minutos (hasta un máximo de 3 a 5 dosis) ${ }^{15}$. Hay que tener precaución al asociarlo con el sulfato de magnesio (puede producir mayor hipotensión) y no se debe administrar nifedipino por vía sublingual ${ }^{37}$. El Atenolol, los IECAs y los Bloqueadores de los receptores de angiotensina están contraindicados en el embarazo.

- Uso de corticoides:

- En caso de gestaciones entre 32 a 34 semanas se procederá a administrar corticoides para maduración pulmonar fetal: betametasona 12 mg IM cada 24 horas por 2 dosis; luego de 48 a 72 horas de iniciado el corticoide se culminará la gestación.

- En gestaciones entre las 24 y 31 semanas: se debe administrar corticoides y tratar de dar manejo expectante hasta donde sea posible según estabilidad materno - fetal.

\section{- Solicitar interconsultas:}

- $\mathrm{UCl}$ neonatal en gestaciones menor o igual a 34 semanas.

- UCl materna en todos los casos.

- Culminación del embarazo ${ }^{37}$.

- En gestaciones $>34$ semanas .

- En gestaciones entre 32 a 34 semanas con maduración pulmonar completa. 
- En hipertensión severa no controlada, es decir, si la paciente requiere la necesidad de usar tres veces seguidas (cada 20 minutos) el nifedipino para hipertensión severa; o si en tres ocasiones aisladas en un lapso de 24 horas presenta tres crisis hipertensivas.

- Síntomas persistentes premonitorios de eclampsia.

- Daño de órgano blanco y/ o su progresión.

o Alteración del bienestar fetal 2,12.

- Eclampsia, edema cerebral.

- Síndrome HELLP, coagulación intravascular diseminada.

- Hemorragia cerebral, encefalopatía hipertensiva.

- Insuficiencia renal aguda, edema agudo de pulmón.

- Hematoma subcapsular hepático o rotura hepática.

- Desprendimiento prematuro de placenta.

- En caso de gestaciones < 24 semanas culminar la gestación.

- Vía del parto: En general se acepta que la vía vaginal es preferible a la cesárea, salvo contraindicación obstétrica ${ }^{37}$ y/o compromiso materno-fetal que amerite la culminación inmediata de la gestación. En caso de culminarse la gestación por cesárea, se realizará una incisión MIU con el fin de revisar el aspecto del hígado.

\section{- Control postparto en hospitalización:}

- Monitoreo estricto de funciones vitales: Presión arterial, frecuencia cardiaca, frecuencia respiratoria (en forma horaria las primeras 24 horas en caso de preeclampsia severa).

- Control estricto de laboratorio según evolución.

- En caso de preeclampsia severa usar metildopa en forma horaria.

- Control de la hipertensión severa con nifedipino. Tener en cuenta que la hipertensión, después de una fase inicial de mejoría, puede presentar un empeoramiento entre el $3^{\circ}$ y $6^{\circ}$ día.

- La infusión de $\mathrm{SO} 4 \mathrm{Mg}$ se mantendrá hasta 24 horas post-parto. Si transcurridas las 24 horas persisten signos o síntomas de severidad, se mantendrá por 24 horas más.

- Para el tratamiento del dolor en las post-operadas se indicará tramadol SC las primeras 24 horas, luego de lo cual se indicará paracetamol, no se recomienda el uso de AINES.

- En caso de hipertensión arterial no controlada solicitar interconsulta a cardiología.

- En caso de preeclampsia severa no dar de alta antes de las 72 horas postparto, aunque esté asintomática.

En la actualidad hay controversia entre el procedimiento inicial de tratamiento agresivo, activo o intervencionista y el tratamiento conservador o expectante ${ }^{24,25}$. Sin embargo en el estudio realizado por Vigil De Gracia y colaboradores, Expectant management of severe preeclampsia remote from term: the MEXPRE Latin Study, a randomized, multicenter clinical trial, publicado en el American Journal of Obstetrics and Gynaecology en noviembre del 2013, se concluye que no existe un beneficio neonatal con el manejo expectante de la preeclampsia severa 28-34 semanas; y además agrega que un enfoque conservador puede aumentar el riesgo de desprendimiento prematuro de placenta y recién nacidos pequeños para la edad gestacional. Hay que tener en cuenta que este es el estudio aleatorio de manejo de preeclampsia severa con más pacientes publicado hasta el día de hoy. En el Instituto Nacional Materno Perinatal el manejo de la preeclampsia severa antes de las 34 semanas sigue las conclusiones de este estudio: administrar corticoides para la inducir la maduración pulmonar y terminar la gestación generalmente por cesárea. Este manejo ha permitido mejorar el pronóstico materno perinatal. La información es insuficiente para decidir si es mejor intervenir o implementar una política de conducta expectante en mujeres con una aparición precoz de preeclampsia severa. La falta de recursos humanos y de materiales para supervisar a las mujeres intensivamente durante un período prolongado puede dificultar la implementación de la conducta expectante en lugares con escasos recursos ${ }^{2}$

\section{MANEJO DE LA ECLAMPSIA}

Las convulsiones eclámpticas amenazan la vida y debe considerarse como una urgencia vital, estas pueden producirse antes del parto, durante o después del mismo y puede aparecer aún con niveles de hipertensión no consideradas graves.

El manejo de la eclampsia requiere el control de las convulsiones y prevención de las recurrencias con sulfato de magnesio ${ }^{26}$ a las mismas dosis indicadas en la preeclampsia severa: sulfato de magnesio 4 gramos endovenosos en 20 minutos; el sulfato de magnesio está asociado con reducciones clínicamente significativas en la muerte materna. Es accesible y su administración y control resultan relativamente sencillos. Se puede administrar por vía intramuscular, 2 gr en cada nalga con 0.5 cc de lidocaína $2 \%$, cuando no se dispone de personal con experiencia en la administración intravenosa ${ }^{1,10,}$

Al mismo tiempo se debe realizar la corrección de la hipoxemia materna y/o acidosis, controlar la hipertensión arterial y realizar el monitoreo de funciones vitales maternas. Se deben solicitar exámenes auxiliares: pruebas hepáticas, pruebas renales, perfil de coagulación y hemoglobina; evaluar el bienestar fetal, Se debe coordinar con la unidad de cuidados intensivos materno y con la unidad de cuidados intensivos neonatal, asi como con anestesiología;

Una vez estabilizada la paciente se debe iniciar el proceso de parto, que en la mayoría de veces requiere una cesárea de emergencia. En el post parto la paciente debe continuar su hospitalización en una Unidad de Cuidados Intensivos Materno ${ }^{10}$. 


\section{MANEJO DEL SINDROME DE HELLP}

El Sindrome HELLP es un transtorno severo del estado gestacional y puerperal relacionado con las formas graves de preeclampsia y eclampsia. Algunos autores admiten la posibilidad de que esta noxa exista sin vínculo alguno con preeclampsia. Se discute si se trata de un síndrome o debe identificarse como una entidad con características clínicas propias ${ }^{27}$

Louis Weinstein el 15 de Enero de 1982 publicó los primeros 29 casos diagnosticados en pacientes con preeclampsia y eclampsia con el acrónimo HELLP: H : Hemolisis (anemia microangiopática, incremento de la bilirrubina a predominio indirecto); EL: enzimas hepáticas elevadas; y LP: plaquetas disminuidas.

Requiere internamiento de urgencia en una unidad de cuidados intensivos, estabilizar la condición materna y culminar la gestación en el menor tiempo posible, independientemente de la edad gestacional y que en la mayoría de casos es por cesárea. El manejo expectante no está recomendado ${ }^{1,9}$. Algunos estudios están considerando el uso de corticoides para mejorar el estado materno y fetal, especialmente cuando el recuento plaquetario es muy bajo ${ }^{1,9}$. El síndrome de HELLP se complica muy frecuentemente con insuficiencia respiratoria, cardiaca, hematoma subcapsular hepático o rotura hepática, insuficiencia renal, desprendimiento prematuro de placenta y hemorragia post parto, disfuinción hematológica y disfunción neurológica. La morbilidad materna extrema y mortalidad materna es muy alta ${ }^{27}$.

\section{HEMATOMA SUBCAPSULAR HEPÁTICO Y RUPTURA HEPATICA}

Aberombic ${ }^{28}$ describio' el hematoma subcapsular hepático, que se presenta en 1:45.000-225.000 embarazos, o lo que es lo mismo, en un $1 \%$ de los casos de síndrome de HELLP. La posibilidad de recurrencia en embarazos posteriores oscila entre el $4-27 \%{ }^{29}$.

La sospecha clínica, el diagnóstico rápido y un tratamiento multidisciplinario son fundamentales para mejorar la morbimortalidad de estas pacientes. El tratamiento abarca desde la conducta expectante y seguimiento clínico ${ }^{30}$, la embolización de las arterias hepaticas ${ }^{31} \mathrm{o}$ incluso se propone tratamiento quirúrgico en presencia de inestabilidad hemodinámica, persistencia de sangrado o aumento del dolor ${ }^{32}$.

El tratamiento clásico se basa en el abordaje quirúrgico de la rotura hepática. Pueden ser el packing o la ligadura de las arterias hepáticas, resecciones e incluso trasplantes hepático ${ }^{33}$. En el Instituto Nacional Materno Perinatal se viene usando el empaquetamiento hepático temporal para controlar la hemorragia con colocación de bolsa de Bogotá, que consiste en suturar a la aponeurosis una lámina de plástico para dejar contenido el abdomen, con el objeto de disminuir la tensión de la pared abdominal y evitar el síndrome compartimental ${ }^{34}$.

Se han descrito también técnicas en base al bisturí de argón ${ }^{35}$ y de administración de factor VIla recombinante ${ }^{35}$ para la hemostasia hepática.

Ante la sospecha de un síndrome de HELLP complicado con rotura hepática y la necesidad de practicar una cesárea urgente, se recomienda el abordaje quirúrgico del hígado en el mismo acto. Si, el síndrome de HELLP se desarrolla en el puerperio y tiene un hígado sano, se puede valorar un manejo más conservador mediante la embolización de la arteria hepática común o bien una de sus ramas principales.

Conflictos de interés: Ninguno.

\section{REFERENCIAS BIBLIOGRAFICAS}

1. Report of the American College of Obstetricians and Gynecologists. Task Force on Hypertension in Pregnancy. Hypertension in Pregnancy. Obstetrics \& Gynecology. VOL. 122, NO. 5, NOVEMBER 2013. Pg 1122 - 1131

2. Pacheco J. Peeclampsia/Eclampsia: Reto para el Ginecoobstetra. Acta Médica Peruana. 23(2)2006.Pg. 100 - 111

3. Pacheco J, Wagner P, Williams N, Sánchez S. Enfermedad Hlpertensiva de la gestación. Ginecología y Obstetricia. Segunda Edición. Lima: MAD Corp SA 2006

4. Chirinos J. Incidencia y características de la Enfermedad Hipertensiva en el embarazo: Estudio retrospectivo a nivel del mar y en la altura. Acta Andina. 1995; 4 (1): 25 - 34

5. Dirección General de Epidemiología. Ministerio de Salud. Sistema Nacional de Vigilancia Epidemiológica . 2012.

6. Instituto Nacional Materno Perinatal. Oficina de Estadística e Informática. Información para la red 2013.

7. National High Blood Pressure Education Program. Working Group Report on High Blood Pressure in Pregnancy. National Institute of Health Publication No. 00-3029 Originally Printed 1990. Revised July 2000;Pg 1 - 83

8. American College of Obstetricians and Gynecologists. Hypertension in Pregnancy. 2013. Pg 1 - 89.

9. American College of Obstetricians and Gynecologists. Diagnosis and Management of Preeclampsia and Eclampsia. Practice Bulletin Clinical Management Guidelines For Obstetrician-Gynecologists. Number 33, January 2002; Pg: 159 - 167.

10. Instituto Nacional Materno Perinatal. Guías de Práctica Clínica y de Procedimientos en Obstetricia y Perinatologia. 2014. Pg. $69-83$

11. Chovanian A,Brakis G, Black H,Cushman W. et al. The Seven Report of the Joint National Committee on Prevention, Detection. Evaluation, and Treatment of High Blood Pressure. JAMA 2003: 289 (19).

12. John Bernard. El Laboratorio en el Diagnóstico clínico. Marban Libros. S. L. 2007

13. Maybury Hand Waugh J. Proteinuria in Pregnancy-Just what is significant? Fetal and Maternal Medicine Review. 2004 16: 71-95.)

14. Kuo VS, Koumantakis G and Gallery EDM. Proteinuria and its assessment in normal and hypertensive pregnancy. Am J Obstet Gynecol 1992; 167: 723-728. 
15. Brown MA. Diagnosis and Classification of Preeclampsia and other Hypertensive Disorders of Pregnancy. En: Hypertension in Pregnancy, edited: Belfort MA, Thornton $S$ and Saade GR.New York, Marcel Dekker, 2003: 1-16.

16. Bautista A. Hipertensión y embarazo. Toxemia gravídica. En: Ñáñez $H$, Ruiz Al, eds. Texto de obstetricia y perinatologia Una contribución a la enseñanza del arte,ciencia y tecnologia. Pharmacia Upjohn. 1999. Capítulo 33, 487-524.

17. Federación Latinoamericana de Sociedades de Obstetricia y Ginecología. Módulo de capacitación en Pre-Eclampsia/ Eclampsia. Diciembre 2012. Pg 1 - 53

18. World Health Organization. WHO recommendations for Prevention and treatment of pre-eclampsia and eclampsia. 2011. Pg 1 - 38

19. Royal College of Obstetricians and Gynaecologists. Hypertension in pregnancy: the management of hypertensive disorders during pregnancy. Agosto 2010. Pg 111 - 178.

20. Journal of Obstetrics and Gynaecology Canada. Diagnosis, Evaluation, and Management of the Hypertensive Disorders of Pregnancy. Volume 30, Number 3. March 2008. S1 - S48

21. Sociedad Española de Ginecología y Obstetricia. Guía Práctica de Urgencias en Obstetricia y Ginecología. Hipertensión arterial y gestación. Mayo 2008. Pg. 33 - 36

22. Khan KS. Sulfato de magnesio y otros anticonvulsivos en mujeres con preeclampsia: Comentario de la BSR (última revisión: 8 de septiembre de 2003). Biblioteca de Salud Reproductiva de la OMS; Ginebra: Organización Mundial de la Salud.

23. Jennifer Uzan et al. Preeclampsia fisiopatología, diagnóstico y tratamiento. Vascular Health and Risk Management 2011:7 467-474.

24. Ganzevoort, W, MD, Sibai, B, MD, Temporising versus interventionist management (preterm and at term). Best Practice \& Research Clinical Obstetrics and Gynaecology. Elsevier Ltd. 2011. Pg 463 - 476.

25. Lombaard $\mathrm{H}$ y Pattinson $\mathrm{B}$. Conducta activa versus conducta expectante para la preeclampsia severa antes de término: Comentario de la BSR (última revisión: 5 de agosto de 2004). La Biblioteca de Salud Reproductiva de la OMS; Ginebra: Organización Mundial de la Salud).

26. Atallah AN. Tratamiento anticonvulsivo para la eclampsia: Comentario de la BSR (última revisión: 14 de noviembre de 2003). La Biblioteca de Salud Reproductiva de la OMS; Ginebra: Organización Mundial de la Salud.
27. Reubinoff BE, Schenker IG. HELLP syndrome complicating preeclampsia, eclampsia. Int J Gyneacol Obstet 1991; 36:95102).

28. Aberombic J. Haemorrage of the liver. London Medical Gazette.1984;34:792-4)

29. Rojas G. HELLP syndrome. Critical state. Current concepts. Ginecol Obstet Mex. 1996;64:64-72

30. Pilco P. Ruptured subcapsular hepatic hematoma associated with HELLP syndrome. Rev Gastroenterol Peru'. 2006;26:207-10

31. Rinehart BK. Preeclampsia-associated hepatic hemorrhage and rupture: mode of management related to maternal and perinatal outcome. Obstet Gynecol Survey. 1999;54:196

32. Wilson RH. Postpartum rupture of subcapsular hematoma of the liver. Acta Obstet Gynecol Scandinav. 1992;71:394

33. Reck T. Surgical treatment of HELLP syndrome-associated liver rupture. An update. Eur J Gynecol Reprod Biol. 2001;99:57-65)

34. Shrivastava VK. Argon beam coagulator for treatment of hepatic rupture with hemolysis, elevated liver enzymes, low platelets (HELLP) syndrome. Obstet Gynecol. 2006;107:525-6

35. Dart BW. A novel use of recombinant factor VIla in HELLP associated with spontaneous hepatic rupture and abdominal compartment syndrome. J Trauma. 2004;57:171-174.

36. Sibai, Baha. Etiology and management of postpartum hypertension-preeclampsia. American Journal of Obstetrics \& Gynecology. JUNE 2012. Pg 470 - 475.

37. Ministerio de Salud. Dirección General De Salud De Las Personas. Estrategia Sanitaria Nacional de Salud Sexual y Reproductiva. Guías De Práctica Clínica para la Atención de Emergencias Obstétricas según nivel de Capacidad Resolutiva. Lima; 2007; Pg 53 - 62.

38. Ministerio de Salud. Dirección General de Salud de las Personas. Dirección Ejecutiva de Atención Integral de Salud. Guías Nacionales de Atención Integral de Salud sexual y Reproductiva. Módulo III Emergencias Obstétricas: Lima, 2004. Pg 16 - 20

39. Organización Panamericana de la Salud. Manejo de las complicaciones del embarazo y el parto: Guía para obstetrices y médicos. 2002. Sección II, Pg S39 - S62.

Correspondencia: enriqueguevararios@gmail.com; eguevara@ iemp.gob.pe; enriqueg@pathfinder.org; eguevara@insap.org.pe 